\title{
Serre Dimension of Monoid Algebras
}

\author{
Manoj K. Keshari and Husney Parvez Sarwar \\ Department of Mathematics, IIT Bombay, Powai, Mumbai - 400076, India; \\ keshari@math.iitb.ac.in; mathparvez@gmail.com
}

\begin{abstract}
Let $R$ be a commutative Noetherian ring of dimension $d, M$ a commutative cancellative torsionfree monoid of rank $r$ and $P$ a finitely generated projective $R[M]$-module of rank $t$.

(1) Assume $M$ is $\Phi$-simplicial seminormal. (i) If $M \in \mathcal{C}(\Phi)$, then Serre $\operatorname{dim} R[M] \leq d$. (ii) If $r \leq 3$, then Serre $\operatorname{dim} R[\operatorname{int}(M)] \leq d$.

(2) If $M \subset \mathbb{Z}_{+}^{2}$ is a normal monoid of rank 2 , then Serre dim $R[M] \leq d$.

(3) Assume $M$ is $c$-divisible, $d=1$ and $t \geq 3$. Then $P \cong \wedge^{t} P \oplus R[M]^{t-1}$.

(4) Assume $R$ is a uni-branched affine algebra over an algebraically closed field and $d=1$. Then $P \cong \wedge^{t} P \oplus R[M]^{t-1}$.
\end{abstract}

\section{Introduction}

Throughout rings are commutative Noetherian with 1; projective modules are finitely generated and of constant rank; monoids are commutative cancellative torsion-free; $\mathbb{Z}_{+}$denote the additive monoid of non-negative integers.

Let $A$ be a ring and $P$ a projective $A$-module. An element $p \in P$ is called unimodular, if there exists $\phi \in \operatorname{Hom}(P, A)$ such that $\phi(p)=1$. We say Serre dimension of $A$ (denoted as Serre $\operatorname{dim} A$ ) is $\leq t$, if every projective $A$-module of rank $\geq t+1$ has a unimodular element. Serre dimension of $A$ measures the surjective stabilization of the Grothendieck group $K_{0}(A)$. Serre's problem on the freeness of projective $k\left[X_{1}, \ldots, X_{n}\right]$-modules, $k$ a field, is equivalent to Serre $\operatorname{dim} k\left[X_{1}, \ldots, X_{n}\right]=0$.

After the solution of Serre's problem by Quillen [16] and Suslin [21, many people worked on surjective stabilization of polynomial extension of a ring. Serre [20] proved Serre $\operatorname{dim} A \leq \operatorname{dim} A$, Plumstead [14] proved Serre $\operatorname{dim} A[X] \leq \operatorname{dim} A$, Bhatwadekar-Roy [4] proved Serre $\operatorname{dim} A\left[X_{1}, \ldots, X_{n}\right] \leq \operatorname{dim} A$ and Bhatwadekar-Lindel-Rao [3] proved Serre $\operatorname{dim} A\left[X_{1}, \ldots, X_{n}, Y_{1}^{ \pm 1}, \ldots, Y_{m}^{ \pm 1}\right] \leq \operatorname{dim} A$.

Anderson conjectured an analogue of Quillen-Suslin theorem for monoid algebras over a field which was answered by Gubeladze [8] (see 1.1) as follows.

Theorem 1.1 Let $k$ be a field and $M$ a monoid. Then $M$ is seminormal if and only if all projective $k[M]$-modules are free.

Gubeladze [1] asked the following

Question 1.2 Let $M \subset \mathbb{Z}_{+}^{r}$ be a monoid of rank $r$ with $M \subset \mathbb{Z}_{+}^{r}$ an integral extension. Let $R$ be a ring of dimension $d$. Is Serre $\operatorname{dim} R[M] \leq d$ ? 
We answer Question 1.2 for some class of monoids. Recall that a finitely generated monoid $M$ of rank $r$ is called $\Phi$-simplicial if $M$ can be embedded in $\mathbb{Z}_{+}^{r}$ and the extension $M \subset \mathbb{Z}_{+}^{r}$ is integral (see [10]). A $\Phi$-simplicial monoid is commutative, cancellative and torsion-free.

Definition 1.3 Let $\mathcal{C}(\Phi)$ denote the class of seminormal $\Phi$-simplicial monoids $M \subset \mathbb{Z}_{+}^{r}$ of rank $r$ such that if $\mathbb{Z}_{+}^{r}=\left\{t_{1}^{s_{1}} \ldots t_{r}^{s_{r}} \mid s_{i} \geq 0\right\}$, then for $1 \leq m \leq r, M_{m}=M \cap\left\{t_{1}^{s_{1}} \ldots t_{m}^{s_{m}} \mid s_{i} \geq 0\right\}$ satisfies the following properties: Given a positive integer $c$, there exist integers $c_{i}>c$ for $i=1, \ldots, m-1$ such that for any ring $R$, the automorphism $\eta \in A u t_{R\left[t_{m}\right]}\left(R\left[t_{1}, \ldots, t_{m}\right]\right)$ defined by $\eta\left(t_{i}\right)=t_{i}+t_{m}^{c_{i}}$ for $i=1, \ldots, m-1$, restricts to an $R$-automorphism of $R\left[M_{m}\right]$. It is easy to see that $M_{m} \in \mathcal{C}(\Phi)$ and rank $M_{m}=m$ for $1 \leq m \leq r$.

The following result $(3.4,3.8)$ answers Question 1.2 for monoids in $\mathcal{C}(\Phi)$.

Theorem 1.4 Let $M \subset \mathbb{Z}_{+}^{r}$ be a seminormal $\Phi$-simplicial monoid of rank $r$ and $R$ a ring of dimension $d$.

(1) If $M \in \mathcal{C}(\Phi)$, then Serre $\operatorname{dim} R[M] \leq d$.

(2) If $r \leq 3$, then Serre $\operatorname{dim} R[\operatorname{int}(M)] \leq d$, where $\operatorname{int}(M)=\operatorname{int}\left(\mathbb{R}_{+} M\right) \cap \mathbb{Z}_{+}^{3}$ and $\operatorname{int}\left(\mathbb{R}_{+} M\right)$ is the interior of the cone $\mathbb{R}_{+} M \subset \mathbb{R}^{3}$ with respect to Euclidean topology.

The following result (3.6) follows from (1.4(1)). When $R$ is a field, this result is due to Anderson 1].

Theorem 1.5 Let $R$ be a ring of dimension $d$ and $M \subset \mathbb{Z}_{+}^{2}$ a normal monoid of rank 2 . Then Serre $\operatorname{dim} R[M] \leq d$.

The next result answers Question 1.2 partially for 1-dimensional rings (see 3.13, 3.16). The proof uses the techniques of Kang [12, Roy [17] and Gubeladze's [9]. Let us recall two definitions. (i) A monoid $M$ is called $c$-divisible, where $c>1$ is an integer, if $c X=m$ has a solution in $M$ for all $m \in M$. All $c$-divisible monoids are seminormal. (ii) Let $R$ be a ring, $\bar{R}$ the integral closure of $R$ and $C$ the conductor ideal of $R \subset \bar{R}$. Then $R$ is called uni-branched if for any $\mathfrak{p} \in \operatorname{Spec} R$ containing $C$, there is a unique $\mathfrak{q} \in \operatorname{Spec} \bar{R}$ such that $\mathfrak{q} \cap R=\mathfrak{p}$.

Theorem 1.6 Let $R$ be a ring of dimension $1, M$ a monoid and $P$ a projective $R[M]$-module of rank $r$.

(i) If $M$ is c-divisible and $r \geq 3$, then $P \cong \wedge^{r} P \oplus R[M]^{r-1}$.

(ii) If $R$ is a uni-branched affine algebra over an algebraically closed field, then $P \cong \wedge^{r} P \oplus R[M]^{r-1}$.

If $R$ is a 1-dimensional anodal ring with finite seminormalization, then (1.6)(ii)) is due to Sarwar (18), Theorem 1.2). Note that if $k$ is an algebraically closed field of characteristic 2, then node $k[X, Y] /\left(X^{2}-Y^{2}-Y^{3}\right)$ is not anodal but is uni-branched, by Kang ([12, Example 2).

At the end, we give some applications to minimum number of generators of projective modules. 


\section{Preliminaries}

Let $A$ be a ring and $Q$ an $A$-module. We say $p \in Q$ is unimodular if the order ideal $O_{Q}(p)=$ $\{\phi(p) \mid \phi \in \operatorname{Hom}(Q, A)\}$ equals $A$. The set of all unimodular elements in $Q$ is denoted by $\operatorname{Um}(Q)$. We write $\mathrm{E}_{n}(A)$ for the group generated by set of all $n \times n$ elementary matrices over $A$ and $\operatorname{Um}_{n}(A)$ for $\operatorname{Um}\left(A^{n}\right)$. We denote by Aut ${ }_{A}(Q)$, the group of all $A$-automorphisms of $Q$.

For an ideal $J$ of $A$, we denote by $\mathrm{E}(A \oplus Q, J)$, the subgroup of Aut ${ }_{A}(A \oplus Q)$ generated by all the automorphisms $\Delta_{a \phi}=\left(\begin{array}{ll}1 & a \phi \\ 0 & i d_{Q}\end{array}\right)$ and $\Gamma_{q}=\left(\begin{array}{cc}1 & 0 \\ q & i d_{Q}\end{array}\right)$ with $a \in J, \phi \in Q^{*}$ and $q \in Q$. Further, we shall write $\mathrm{E}(A \oplus Q)$ for $\mathrm{E}(A \oplus Q, A)$. We denote by $\operatorname{Um}(A \oplus Q, J)$ the set of all $(a, q) \in \operatorname{Um}(A \oplus Q)$ with $a \in 1+J$ and $q \in J Q$.

We state some results of Lindel [13] for later use.

Proposition 2.1 (Lindel [13], 1.1) Let $A$ be a ring and $Q$ an A-module. Let $Q_{s}$ be free of rank $r$ for some $s \in A$. Then there exist $p_{1}, \ldots, p_{r} \in Q, \phi_{1}, \ldots, \phi_{r} \in Q^{*}$ and $t \geq 1$ such that following holds:

(i) $0::_{A} s^{\prime} A=0:_{A} s^{2} A$, where $s^{\prime}=s^{t}$.

(ii) $s^{\prime} Q \subset F$ and $s^{\prime} Q^{*} \subset G$, where $F=\sum_{i=1}^{r} A p_{i} \subset Q$ and $G=\sum_{i=1}^{r} A \phi_{i} \subset Q^{*}$.

(iii) the matrix $\left(\phi_{i}\left(p_{j}\right)\right)_{1 \leq i, j \leq r}=$ diagonal $\left(s^{\prime}, \ldots, s^{\prime}\right)$. We say $F$ and $G$ are $s^{\prime}$-dual submodules of $Q$ and $Q^{*}$ respectively.

Proposition 2.2 (Lindel [13], 1.2, 1.3) Let $A$ be a ring and $Q$ an A-module. Assume $Q_{s}$ is free of rank $r$ for some $s \in A$. Let $F$ and $G$ be $s$-dual submodules of $Q$ and $Q^{*}$ respectively. Then

(i) for $p \in Q$, there exists $q \in F$ such that ht $\left(O_{Q}(p+s q) A_{s}\right) \geq r$.

(ii) If $Q$ is projective $A$-module and $\bar{p} \in \operatorname{Um}(Q / s Q)$, then there exists $q \in F$ such that ht $\left(O_{Q}(p+\right.$ $s q)) \geq r$.

Proposition 2.3 (Lindel [13], 1.6) Let $Q$ be a module over a positively graded ring $A=\oplus_{i \geq 0} A_{i}$ and $Q_{s}$ be free for some $s \in R=A_{0}$. Let $T \subseteq A$ be a multiplicatively closed set of homogeneous elements. Let $p \in Q$ be such that $p_{T(1+s R)} \in \operatorname{Um}\left(Q_{T(1+s R)}\right)$ and $s \in \operatorname{rad}\left(O_{Q}(p)+A_{+}\right)$, where $A_{+}=\oplus_{i \geq 1} A_{i}$. Then there exists $p^{\prime} \in p+s A_{+} Q$ such that $p_{T}^{\prime} \in \operatorname{Um}\left(Q_{T}\right)$.

Proposition 2.4 (Lindel [13], 1.8) Under the assumptions of [2.3), let $p \in Q$ be such that $O_{Q}(p)+$ $s A_{+}=A$ and $A / O_{Q}(p)$ is an integral extension of $R /\left(R \cap O_{Q}(p)\right)$. Then there exists $p^{\prime} \in \operatorname{Um}(Q)$ with $p^{\prime}-p \in s A_{+} Q$.

The following result is due to Amit Roy (17], Proposition 3.4).

Proposition 2.5 Let $A, B$ be two rings with $f: A \rightarrow B$ a ring homomorphism. Let $s \in A$ be nonzerodivisor such that $f(s)$ is a non-zerodivisor in B. Assume that we have the following cartesian square.

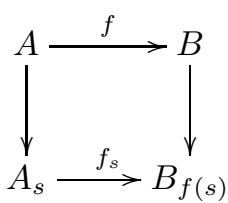


Further assume that $\mathrm{SL}_{r}\left(B_{f(s)}\right)=\mathrm{E}_{r}\left(B_{f(s)}\right)$ for some $r>0$. Let $P$ and $Q$ be two projective $A$-modules of rank $r$ such that $(i) \wedge^{r} P \cong \wedge^{r} Q$, (ii) $P_{s}$ and $Q_{s}$ are free over $A_{s}$, (iii) $P \otimes_{A} B \cong Q \otimes_{A} B$ and $Q \otimes{ }_{A} B$ has a unimodular element. Then $P \cong Q$.

Definition 2.6 (see [10, Section 6) Let $R$ be a ring and $M$ a $\Phi$-simplicial monoid of rank $r$. Fix an integral extension $M \hookrightarrow \mathbb{Z}_{+}^{r}$. Let $\left\{t_{1}, \ldots, t_{r}\right\}$ be a free basis of $\mathbb{Z}_{+}^{r}$. Then $M$ can be thought of as a monoid consisting of monomials in $t_{1}, \ldots, t_{r}$.

For $x=t_{1}^{a_{1}} \ldots t_{r}^{a_{r}}$ and $y=t_{1}^{b_{1}} \ldots t_{r}^{b_{r}}$ in $\mathbb{Z}_{+}^{r}$, define $x$ is lower than $y$ if $a_{i}<b_{i}$ for some $i$ and $a_{j}=b_{j}$ for $j>i$. In particular, $t_{i}$ is lower than $t_{j}$ if and only if $i<j$.

For $f \in R[M]$, define the highest member $H(f)$ of $f$ as am, where $f=a m+a_{1} m_{1}+\ldots+a_{k} m_{k}$ with $m, m_{i} \in M, a \in R \backslash\{0\}, a_{i} \in R$ and each $m_{i}$ is strictly lower than $m$ for $1 \leq i \leq k$.

An element $f \in R\left[\mathbb{Z}_{+}^{r}\right]$ is called monic if $H(f)=a t_{r}^{s}$, where $a \in R$ is a unit and $s>0$. An element $f \in R[M]$ is said to be monic if $f$ is monic in $R\left[\mathbb{Z}_{+}^{r}\right]$ via the embedding $R[M] \hookrightarrow R\left[\mathbb{Z}_{+}^{r}\right]$.

Define $M_{0}$ to be the submonoid $\left\{t_{1}^{s_{1}} \ldots t_{r-1}^{s_{r-1}} \mid s_{i} \geq 0\right\} \cap M$ of $M$. Clearly $M_{0}$ is finitely generated as $M$ is finitely generated. Also $M_{0} \hookrightarrow \mathbb{Z}_{+}^{r-1}$ is integral. Hence $M_{0}$ is $\Phi$-simplicial. Further, if $M$ is seminormal, then $M_{0}$ is seminormal.

Grade $R[M]$ as $R[M]=R\left[M_{0}\right] \oplus A_{1} \oplus A_{2} \oplus \ldots$, where $A_{i}$ is the $R\left[M_{0}\right]$-module generated by the monomials $t_{1}^{s_{1}} \ldots t_{r-1}^{s_{r-1}} t_{r}^{i} \in M$. For an ideal $I$ in $R[M]$, define its leading coefficient ideal $\lambda(I)$ as $\{a \in R \mid \exists f \in I$ with $H(f)=a m$ for some $m \in M\}$.

Lemma 2.7 ([10], Lemma 6.5) Let $R$ be a ring and $M \subset \mathbb{Z}_{+}^{r}$ a $\Phi$-simplicial monoid. If $I \subseteq R[M]$ is an ideal, then ht $(\lambda(I)) \geq \mathrm{ht}(I)$, where $\lambda(I)$ is defined in (2.6).

\section{Main Theorem}

This section contains main results stated in the introduction. We also give some examples of monoids in $C(\Phi)$.

\subsection{Over $C(\Phi)$ class of monoids}

Lemma 3.1 Let $R$ be a ring and $M \subset \mathbb{Z}_{+}^{r}$ a monoid in $\mathcal{C}(\Phi)$ of rank $r$. Let $f \in R[M] \subset R\left[\mathbb{Z}_{+}^{r}\right]=$ $R\left[t_{1}, \ldots, t_{r}\right]$ with $H(f)=u t_{1}^{s_{1}} \ldots t_{r}^{s_{r}}$ for some unit $u \in R$. Then there exist $\eta \in \operatorname{Aut}_{R}(R[M])$ such that $\eta(f)$ is a monic polynomial in $t_{r}$.

Proof Since $M \in C(\Phi)$, we can choose positive integers $c_{1}, \ldots, c_{r-1}$ such that the automorphism $\eta \in A u t_{R\left[t_{r}\right]} R\left[t_{1} \ldots, t_{r}\right]$ defined by $\eta\left(t_{i}\right)=t_{i}+t_{r}^{c_{i}}$ for $i=1, \ldots, r-1$, restricts to an automorphism of $R[M]$ and such that $\eta(f)$ is a monic polynomial in $t_{r}$.

Lemma 3.2 Let $R$ be a ring of dimension $d$ and $M \subset \mathbb{Z}_{+}^{r}$ a monoid in $\mathcal{C}(\Phi)$ of rank $r$. Let $P$ be a projective $R[M]$-module of rank $>d$. Write $R[M]=R\left[M_{0}\right] \oplus A_{1} \oplus A_{2} \ldots$, as defined in (2.6) and 
$A_{+}=A_{1} \oplus A_{2} \oplus \ldots$ an ideal of $R[M]$. Assume that $P_{s}$ is free for some $s \in R$ and $P / s A_{+} P$ has a unimodular element. Then the natural map $\operatorname{Um}(P) \rightarrow \mathrm{Um}\left(P / s A_{+} P\right)$ is surjective. In particular, $P$ has a unimodular element.

Proof Write $A=R[M]$. Since every unimodular element of $P / s A_{+} P$ can be lifted to a unimodular element of $P_{1+s A_{+}}$, if $s$ is nilpotent, then elements of $1+s A_{+}$are units in $A$ and we are done. Therefore, assume that $s$ is not nilpotent.

Let $p \in P$ be such that $\bar{p} \in \operatorname{Um}\left(P / s A_{+} P\right)$. Then $O_{P}(p)+s A_{+}=A$. Hence $O_{P}(p)$ contains an element of $1+s A_{+}$. Choose $g \in A_{+}$such that $1+s g \in O_{P}(p)$. Applying (2.2) with $s g$ in place of $s$, we get $q \in F \subset P$ such that ht $\left(O_{P}(p+s g q)\right)>d$. Since $p+s g q$ is a lift of $\bar{p}$, replacing $p$ by $p+s g q$, we may assume that ht $\left(O_{P}(p)\right)>d$. By (2.7), we get ht $\left(\lambda\left(O_{P}(p)\right)\right) \geq$ ht $\left(O_{P}(p)\right)>d$. Since $\lambda\left(O_{P}(p)\right)$ is an ideal of $R$, we get $1 \in \lambda\left(O_{P}(p)\right)$. Hence there exists $f \in O_{P}(p)$ such that the coefficient of $H(f)$ (highest member of $f$ ) is a unit.

Suppose $H(f)=u t_{1}^{s_{1}} \ldots t_{r}^{s_{r}}$ with $u$ a unit in $R$. Since $M \in \mathcal{C}(\Phi)$, by (3.1), there exists $\alpha \in$ Aut ${ }_{R}(R[M])$ such that $\alpha(f)$ is monic in $t_{r}$. Thus we may assume that $O_{P}(p)$ contains a monic polynomial in $t_{r}$. Hence $A / O_{P}(p)$ is an integral extension of $R\left[M_{0}\right] /\left(O_{P}(p) \cap R\left[M_{0}\right]\right)$ and $\bar{p} \in$ $\mathrm{Um}\left(P / s A_{+} P\right)$. By (2.4), there exists $p^{\prime} \in \operatorname{Um}(P)$ such that $p^{\prime}-p \in s A_{+} P$. This means $p^{\prime} \in \operatorname{Um}(P)$ is a lift of $\bar{p}$. This proves the result.

Remark 3.3 In (3.2), we do not need the monoid $M$ to be seminormal.

The next result proves $[1.4(1))$.

Theorem 3.4 Let $R$ be a ring of dimension d and $M$ a monoid in $\mathcal{C}(\Phi)$ of rank $r$. If $P$ is a projective $R[M]$-module of rank $r^{\prime} \geq d+1$, then $P$ has a unimodular element. In other words, Serre dim $R[M] \leq d$.

Proof We can assume that the ring is reduced with connected spectrum. If $d=0$, then $R$ is a field. Since $M$ is seminormal, projective $R[M]$-modules are free, by (1.1). If $r=0$, then $M=0$ and we are done by Serre [20]. Assume $d, r \geq 1$ and use induction on $d$ and $r$ simultaneously.

If $S$ is the set of all non-zerodivisor of $R$, then $\operatorname{dim} S^{-1} R=0$ and so $S^{-1} P$ is free $S^{-1} R[M]$-module $\left(d=0\right.$ case). Choose $s \in S$ such that $P_{s}$ is free. Consider the ring $R[M] /(s R[M])=(R / s R)[M]$. Since $\operatorname{dim} R / s R=d-1$, by induction on $d, \operatorname{Um}(P / s P)$ is non-empty.

Write $R[M]=R\left[M_{0}\right] \oplus A_{1} \oplus A_{2} \ldots$, as defined in (2.6) and $A_{+}=A_{1} \oplus A_{2} \oplus \ldots$ an ideal of $R[M]$. Note that $M_{0} \in \mathcal{C}(\Phi)$ and rank $M_{0}=r-1$. Since $R[M] / A_{+}=R\left[M_{0}\right]$, by induction on $r, \operatorname{Um}\left(P / A_{+} P\right)$ is non-empty. Write $A=R[M]$ and consider the following fiber product diagram

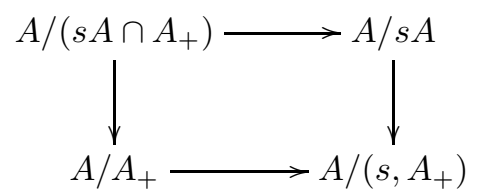


If $B=R / s R$, then $A /\left(s, A_{+}\right)=B\left[M_{0}\right]$. Let $u \in \operatorname{Um}\left(P / A_{+} P\right)$ and $v \in \operatorname{Um}(P / s P)$. Let $\bar{u}$ and $\bar{v}$ denote the images of $u$ and $v$ in $P /\left(s, A_{+}\right) P$. Write $P /\left(s, A_{+}\right) P=B\left[M_{0}\right] \oplus P_{0}$, where $P_{0}$ is some projective $B\left[M_{0}\right]$-module of rank $=r^{\prime}-1$. Note that $\operatorname{dim}(B)=d-1$ and $\bar{u}, \bar{v}$ are two unimodular elements in $B\left[M_{0}\right] \oplus P_{0}$.

Case 1. Assume $\operatorname{rank}\left(P_{0}\right) \geq \max \{2, d\}$. Then by ([6], Theorem 4.5), there exists $\sigma \in \mathrm{E}\left(B\left[M_{0}\right] \oplus\right.$ $\left.P_{0}\right)$ such that $\sigma(\bar{u})=\bar{v}$. Lift $\sigma$ to an element $\sigma_{1} \in \mathrm{E}\left(P / A_{+} P\right)$ and write $\sigma_{1}(u)=u_{1} \in \operatorname{Um}\left(P / A_{+} P\right)$. Then images of $u_{1}$ and $v$ are same in $P /\left(s, A_{+}\right) P$. Patching $u_{1}$ and $v$ over $P /\left(s, A_{+}\right) P$ in the above fiber product diagram, we get an element $p \in \operatorname{Um}\left(P /\left(s A \cap A_{+}\right) P\right)$.

Note $s A \cap A_{+}=s A_{+}$. We have $P_{s}$ is free and $P / s A_{+} P$ has a unimodular element. Use (3.2), to conclude that $P$ has a unimodular element.

Case 2. Now we consider the remaining case, namely $d=1$ and $\operatorname{rank}(P)=2$. Since $B=R / s R$ is 0 dimensional, projective modules over $B\left[M_{0}\right]$ and $B[M]$ are free, by (1.1). In particular, $P / s P$ and $P /\left(s, A_{+}\right) P$ are free modules of rank 2 over the rings $B[M]$ and $B\left[M_{0}\right]$ respectively. Consider the same fiber product diagram as above.

Since any two unimodular elements in $\mathrm{Um}_{2}\left(B\left[M_{0}\right]\right)$ are connected by an element of $\mathrm{GL}_{2}\left(B\left[M_{0}\right]\right)$. Further $B\left[M_{0}\right]$ is a subring of $B[M]=A / s A$. Hence the natural map $\mathrm{GL}_{2}(B[M]) \rightarrow \mathrm{GL}_{2}\left(B\left[M_{0}\right]\right)$ is surjective. Hence any automorphism of $P /\left(s, A_{+}\right) P$ can be lifted to an automorphism of $P / s P$. By same argument as above, patching unimodular elements of $P / s P$ and $P / A_{+} P$, we get a unimodular element in $P /\left(s A \cap A_{+}\right) P$. Since $s A \cap A_{+}=s A_{+}$and $P / s A_{+} P$ has a unimodular element, by (3.2), $P$ has a unimodular element. This completes the proof.

Example 3.5 (1) If $M$ is a $\Phi$-simplicial normal monoid of rank 2, then $M \in \mathcal{C}(\Phi)$. To see this, by ([10, Lemma 1.3), $M \cong\left(\alpha_{1}, \alpha_{2}\right) \cap \mathbb{Z}_{+}^{2}$, where $\alpha_{1}=(a, b)$ and $\alpha_{2}=(0, c)$ and $\left(\alpha_{1}, \alpha_{2}\right)$ is the group generated by $\alpha_{1}$ and $\alpha_{2}$. It is easy to see that $M \cong\left(\left(1, a_{1}\right),\left(0, a_{2}\right)\right) \cap \mathbb{Z}_{+}^{2}$, where $\operatorname{gcd}(b, c)=g$ and $a_{1}=b / g, a_{2}=c / g$. Hence $M \in \mathcal{C}(\Phi)$.

(2) If $M \subset \mathbb{Z}_{+}^{2}$ is a finitely generated rank 2 normal monoid, then it is easy to see that $M$ is $\Phi$-simplicial. Hence $M \in \mathcal{C}(\Phi)$ by (1).

(3) If $M$ is a rank 3 normal quasi-truncated or truncated monoid (see [10, Definition 5.1), then $M \in \mathcal{C}(\Phi)$. To see this, by (10, Lemma 6.6), $M$ satisfies properties of (1.3). Further, $M_{0}$ is a $\Phi$-simplicial normal monoid of rank 2. By $(1), M_{0} \in \mathcal{C}(\Phi)$.

Corollary 3.6 Let $R$ be a ring of dimension $d$ and $M \subset \mathbb{Z}_{+}^{2}$ a normal monoid of rank 2. Then Serre $\operatorname{dim} R[M] \leq d$.

Proof If $M$ is finitely generated, then result follows from (3.5)(2)) and (3.4).

If $M$ is not finitely generated, then write $M$ as a filtered union of finitely generated submonoids, say $M=\cup_{\lambda \in I} M_{\lambda}$. Since $M$ is normal, the integral closure $\bar{M}_{\lambda}$ of $M_{\lambda}$ is contained in $M$. Hence $M=\cup_{\lambda \in I} \bar{M}_{\lambda}$. By ([5], Proposition 2.22), $\bar{M}_{\lambda}$ is finitely generated. If $P$ is a projective $R[M]$-module, then $P$ is defined over $R\left[\bar{M}_{\lambda}\right]$ for some $\lambda \in I$ as $P$ is finitely generated. Now the result follows from (3.5) (2)) and (3.4). 
The following result follows from (3.5) (3)) and (3.4).

Corollary 3.7 Let $R$ be a ring of dimension $d$ and $M$ a truncated or normal quasi-truncated monoid of rank $\leq 3$. Then Serre dim $R[M] \leq d$.

Now we prove $(1.4(2))$.

Proposition 3.8 Let $R$ be a ring of dimension $d$ and $M$ a $\Phi$-simplicial seminormal monoid of rank $\leq 3$. Then Serre $\operatorname{dim} R[\operatorname{int}(M)] \leq d$.

Proof Recall that $\operatorname{int}(M)=\operatorname{int}\left(\mathbb{R}_{+} M\right) \cap \mathbb{Z}_{+}^{3}$. Let $P$ be a projective $R[\operatorname{int}(M)]$-module of rank $\geq d+1$. Since $M$ is seminormal, by ([5], Proposition 2.40), $\operatorname{int}(M)=\operatorname{int}(\bar{M})$, where $\bar{M}$ is the normalization of $M$. Since normalization of a finitely generated monoid is finitely generated (see [5], Proposition 2.22), $\bar{M}$ is a $\Phi$-simplicial normal monoid. By ([10], Theorem 3.1), $\operatorname{int}(M)=\operatorname{int}(\bar{M})$ is a filtered union of truncated (normal) monoids (see [10, Definition 2.2). Since $P$ is finitely generated, we get $P$ is defined over $R[N]$, where $N \subset \operatorname{int}(M)$ is a truncated monoid. By (3.7), Serre $\operatorname{dim} R[N] \leq d$. Hence $P$ has a unimodular element. Therefore Serre $\operatorname{dim} R[\operatorname{int}(M)] \leq d$.

Assumptions: In the following examples, $R$ is a ring of dimension $d$, Monoid operations are written multiplicatively and $K(M)$ denotes the group of fractions of monoid $M$.

Example 3.9 For $n>0$, consider the monoid $M \subset \mathbb{Z}_{+}^{r}$ generated by $\left\{t_{1}^{i_{1}} t_{2}^{i_{2}} \ldots t_{r}^{i_{r}} \mid \sum i_{j}=n\right\}$. Then $M$ is a $\Phi$-simplicial normal monoid. For integers $c_{i}=n k_{i}+1, k_{i}>0$ and $i=1, \ldots, r-1$, consider $\eta \in \operatorname{Aut}_{R\left[t_{r}\right]}\left(R\left[t_{1}, \ldots, t_{r}\right]\right)$ defined by $t_{i} \mapsto t_{i}+t_{r}^{c_{i}}$ for $i=1, \ldots, r-1$.

A typical monomial in the expansion of $\eta\left(t_{1}^{i_{1}} \ldots t_{r-1}^{i_{r}-1} t_{r}^{i_{r}}\right)=\left(t_{1}+t_{r}^{c_{1}}\right)^{i_{1}} \ldots\left(t_{r-1}+t_{r}^{c_{r-1}}\right)^{i_{r-1}} t_{r}^{i_{r}}$ will look like $\left.\left(t_{1}^{i_{1}-l_{1}} t_{r}^{c_{1} l_{1}}\right) \ldots\left(t_{r-1}^{i_{r-1}-l_{r-1}} t_{r}^{c_{r-1} l_{r-1}}\right) t_{r}^{i_{r}}=\left(t_{1}^{i_{1}-l_{1}} \ldots t_{r-1}^{i_{r-1}-l_{r-1}} t_{r}^{l_{1}+\ldots+l_{r-1}+i_{r}}\right) t_{r}^{n\left(k_{1} l_{1}+\ldots+k_{r-1} l_{r-1}\right.}\right)$ which belong to $M$. So $\eta(R[M]) \subset R[M]$. Similarly, $\eta^{-1}(R[M]) \subset R[M]$. Hence $\eta$ restricts to an $R$-automorphism of $R[M]$. Therefore $\eta$ satisfies the property of (1.3) for $M$. It is easy to see that $M_{m}=M \cap\left\{t_{1}^{s_{1}} \ldots t_{m}^{s_{m}} \mid s_{i} \geq 0\right\}$ for $1 \leq m \leq r-1$ also satisfy this property. Hence $M \in \mathcal{C}(\Phi)$. By (3.4), Serre $\operatorname{dim} R[M] \leq d$.

Example 3.10 Let $M$ be a $\Phi$-simplicial monoid generated by monomials $t_{1}^{2}, t_{2}^{2}, t_{3}^{2}, t_{1} t_{3}, t_{2} t_{3}$. For integers $c_{j}=2 k_{j}-1$ with $k_{j}>1$, consider the automorphism $\eta \in \operatorname{Aut}_{R\left[t_{3}\right]}\left(R\left[t_{1}, t_{2}, t_{3}\right]\right)$ defined by $t_{j} \mapsto t_{j}+t_{3}^{c_{j}}$ for $j=1,2$. Then it is easy to see that $\eta$ restricts to an automorphism of $R[M]$.

We claim that $M$ is seminormal but not normal. For this, let

$$
z=\left(t_{3}^{2}\right)^{-1}\left(t_{1} t_{3}\right)\left(t_{2} t_{3}\right)=t_{1} t_{2} \in K(M) \backslash M, \text { but } \mathrm{z}^{2} \in \mathrm{M},
$$

showing that $M$ is not normal. For seminormality, let

$$
z=\left(t_{1}^{2}\right)^{\alpha_{1}}\left(t_{2}^{2}\right)^{\alpha_{2}}\left(t_{3}^{2}\right)^{\alpha_{3}}\left(t_{1} t_{3}\right)^{\alpha_{4}}\left(t_{2} t_{3}\right)^{\alpha_{5}} \in K(M) \text { with } \alpha_{i} \in \mathbb{Z} \text { and } \mathrm{z}^{2}, \mathrm{z}^{3} \in \mathrm{M} \text {. }
$$


We may assume that $0 \leq \alpha_{4}, \alpha_{5} \leq 1$. Now $z^{2} \in M \Rightarrow \alpha_{1}, \alpha_{2} \geq 0$ and $2 \alpha_{3}+\alpha_{4}+\alpha_{5} \geq 0$. If $\alpha_{3}<0$, then $\alpha_{4}=\alpha_{5}=1$ and $\alpha_{3}=-1$. In this case, $z^{3}=\left(t_{1}^{2 \alpha_{1}+1} t_{2}^{2 \alpha_{2}+1}\right)^{3} \notin M$, a contradiction. Therefore $\alpha_{3} \geq 0$ and $z \in M$. Hence $M$ is seminormal. It is easy to see that $M \in \mathcal{C}(\Phi)$. By (3.4), Serre dim $R\left[t_{1}^{2}, t_{2}^{2}, t_{3}^{2}, t_{1} t_{3}, t_{2} t_{3}\right] \leq d$.

Remark 3.11 (1) Let $R$ be a ring and $P$ a projective $R$-module of rank $\geq 2$. Let $\bar{R}$ be the seminormalization of $R$. It follows from arguments in Bhatwadekar (2], Lemma 3.1) that $P \otimes_{R} \bar{R}$ has a unimodular element if and only if $P$ has a unimodular element.

(2) Assume $R$ is a ring of dimension $d$ and $M \in \mathcal{C}(\Phi)$. Let $\bar{M}$ be the seminormalization of $M$. If $\bar{M}$ is in $C(\Phi)$, then Serre $\operatorname{dim} R[M] \leq \max \{1, d\}$, using ([2] and 3.4 ).

(3) Let $(R, \mathfrak{m}, K)$ be a regular local ring of dimension $d$ containing a field $k$ such that either char $k=0$ or char $k=p$ and tr-deg $K / \mathbb{F}_{p} \geq 1$. Let $M$ be a seminormal monoid. Then, using Popescu ([15], Theorem 1) and Swan ([23], Theorem 1.2), we get Serre $\operatorname{dim} R[M]=0$. If $M$ is not seminormal, then Serre $\operatorname{dim} R[M]=1$ using ([1], 2] and [23]).

Example 3.12 For a monoid $M, \bar{M}$ denotes the seminormalization of $M$.

1. Let $M \subset \mathbb{Z}_{+}^{2}$ be a $\Phi$-simplicial monoid generated by $t_{1}^{n}, t_{1} t_{2}, t_{2}^{n}$, where $n \in \mathbb{N}$. We claim that $M$ is normal. To see this, let $z=t_{1}^{i} t_{2}^{j}=\left(t_{1}^{n}\right)^{p}\left(t_{1} t_{2}\right)^{q}\left(t_{2}^{n}\right)^{r} \in K(M)$ with $p, q, r \in \mathbb{Z}$ such that $z^{t} \in M$ for some $t>0$. Then $i, j \geq 0$. We need to show that $z \in M$. We may assume that $0 \leq q<n$. Since $i, j \geq 0$, we get $p, r \geq 0$. Thus $z \in M$ and $M$ is normal. Hence, by (3.6), Serre $\operatorname{dim} R\left[t_{1}^{n}, t_{1} t_{2}, t_{2}^{n}\right] \leq d$.

2. The monoid $M \subset \mathbb{Z}_{+}^{2}$ generated by $t_{1}^{2}, t_{1} t_{2}^{2}, t_{2}^{2}$ is seminormal but not normal. For this, let $z=\left(t_{1} t_{2}^{2}\right)\left(t_{2}^{2}\right)^{-1}=t_{1} \in K(M) \backslash M$. Then $z^{2} \in M$ showing that $M$ is not normal. For seminormality, let $z=\left(t_{1}^{2}\right)^{\alpha}\left(t_{1} t_{2}^{2}\right)^{\beta}\left(t_{2}^{2}\right)^{\gamma} \in K(M)$ with $\alpha, \beta, \gamma \in \mathbb{Z}$ be such that $z^{2}, z^{3} \in M$. We may assume $0 \leq \beta \leq 1$. If $\beta=0$, then $\alpha, \gamma \geq 0$ and hence $z \in M$. If $\beta=1$, then $z^{2} \in M$ implies $\alpha \geq 0$ and $\gamma+1 \geq 0$. If $\gamma=-1$, then $z^{3}=\left(t_{1}\right)^{6 \alpha+3} \notin M$, a contradiction. Hence $\gamma \geq 0$, proving that $z \in M$ and $M$ is seminormal. It is easy to see that $M \in \mathcal{C}(\Phi)$. Therefore, by (3.4), Serre $\operatorname{dim} R\left[t_{1}^{2}, t_{1} t_{2}^{2}, t_{2}^{2}\right] \leq d$.

3. Let $M$ be a monoid generated by $\left(t_{1}^{2}, t_{1} t_{2}^{j}, t_{2}^{2}\right)$, where $j \geq 3$. Then $M$ is not seminormal. For this, if $z=\left(t_{1} t_{2}^{j}\right)\left(t_{2}^{2}\right)^{-1}=t_{1} t_{2}^{j-2} \in K(M) \backslash M$, then $z^{2}=t_{1}^{2} t_{2}^{2(j-2)}$ and $z^{3}=\left(t_{1}^{2}\right)\left(t_{1} t_{2}^{j}\right)\left(t_{2}^{2 j-6}\right)$ are in $M$, showing that $M$ is not seminormal.

If $j=3$, then observe that $t_{1} t_{2}$ belongs to $\bar{M}$. Since the monoid generated by $t_{1}^{2}, t_{1} t_{2}, t_{2}^{2}$ is normal, we get that $\bar{M}$ is generated by $t_{1}^{2}, t_{1} t_{2}, t_{2}^{2}$. Hence Serre $\operatorname{dim} R[\bar{M}] \leq d$ by (1) above.

Observe that if $j$ is odd, then $\bar{M}=\left(t_{1}^{2}, t_{1} t_{2}, t_{2}^{2}\right)$ and if $j$ is even, then $\bar{M}=\left(t_{1}^{2}, t_{1} t_{2}^{2}, t_{2}^{2}\right)$. So Serre $\operatorname{dim} R[\bar{M}] \leq d$ by $(1,2)$ above.

In both cases, applying $[3.11(1))$, we get Serre $\operatorname{dim} R[M] \leq \max \{1, d\}$. 
4. Let $M$ be a monoid generated by $\left(t_{1}^{3}, t_{1} t_{2}^{2}, t_{2}^{3}\right)$ Then $M$ is not seminormal. For this, let $z=$ $\left(t_{1} t_{2}^{2}\right)^{2} t_{2}^{-3} \in K(M) \backslash M$. Then $z^{2}=t_{1}^{3}\left(t_{1} t_{2}^{2}\right) \in M$ and $z^{3}=t_{1}^{6} t_{2}^{3} \in M$. Hence seminormalization of $M$ is $\bar{M}=\left(t_{1}^{3}, t_{1}^{2} t_{2}, t_{1} t_{2}^{2}, t_{2}^{3}\right)$. By (3.9), Serre $\operatorname{dim} R[\bar{M}] \leq d$. Therefore, applying (3.11(1)), we get Serre $\operatorname{dim} R[M] \leq \max \{1, d\}$.

\subsection{Monoid algebras over 1-dimensional rings}

The following result proves $(1.6(\mathrm{i}))$.

Theorem 3.13 Let $R$ be a ring of dimension 1 and $M$ a c-divisible monoid. If $P$ is a projective $R[M]$-module of rank $r \geq 3$, then $P \cong \wedge^{r} P \oplus R[M]^{r-1}$.

Proof If $R$ is normal, then we are done by Swan 23. Assume $R$ is not normal.

Case 1. Assume $R$ has finite normalization. Let $\bar{R}$ be the normalization of $R$ and $C$ the conductor ideal of the extension $R \subset \bar{R}$. Then ht $C=1$. Hence $R / C$ and $\bar{R} / C$ are zero dimensional rings. Consider the following fiber product diagram

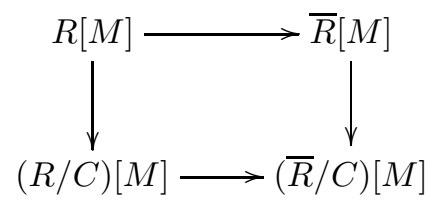

If $P^{\prime}=\wedge^{r} P \oplus R[M]^{r-1}$, then by Swan [23], $P \otimes \bar{R}[M] \cong \wedge^{r}(P \otimes \bar{R}[M]) \oplus \bar{R}[M]^{r-1} \cong P^{\prime} \otimes \bar{R}[M]$. By Gubeladze [8], $P / C P$ and $P^{\prime} / C P^{\prime}$ are free $(R / C)[M]$-modules. Further, $\mathrm{SL}_{r}((\bar{R} / C)[M])=$ $\mathrm{E}_{r}((\bar{R} / C)[M])$ for $r \geq 3$, by Gubeladze [9]. Now using standard arguments of fiber product diagram, we get $P \cong P^{\prime}$.

Case 2. Now $R$ need not have finite normalization. We may assume $R$ is a reduced ring with connected spectrum. Let $S$ be the set of all non-zerodivisors of $R$. By $\left[8, S^{-1} P\right.$ is a free $S^{-1} R[M]$ module. Choose $s \in S$ such that $P_{s}$ is a free $R_{s}[M]$-module.

Now we follow the arguments of Roy ([17, Theorem 4.1). Let $\hat{R}$ denote the $s$-adic completion $R$. Then $\hat{R}_{\text {red }}$ has a finite normalization. Consider the following fiber product diagram

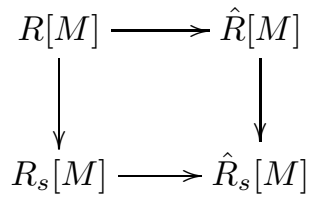

Since $\hat{R}_{s}$ is a zero dimensional ring, by [9], $\mathrm{SL}_{r}\left(\hat{R}_{s}[M]\right)=\mathrm{E}_{r}\left(\hat{R}_{s}[M]\right)$ for $r \geq 3$. If $P^{\prime}=\wedge^{r} P \oplus R[M]^{r-1}$, then $P_{s}$ and $P_{s}^{\prime}$ are free $R_{s}[M]$-modules and by Case $1, P \otimes \hat{R}[M] \cong P^{\prime} \otimes \hat{R}[M]$. By (2.5), $P \cong P^{\prime}$. This completes the proof.

The following result is due to Kang ([12, Lemma 7.1 and Remark). 
Lemma 3.14 Let $R$ be a 1-dimensional uni-branched affine algebra over an algebraically closed field, $\bar{R}$ the normalization of $R$ and $C$ the conductor ideal of the extension $R \subset \bar{R}$. Then $\bar{R} / C=R / C+$ $a_{1} R / C+\cdots+a_{m} R / C$, where $a_{i} \in \sqrt{C}$ the radical ideal of $C$ in $\bar{R}$.

Lemma 3.15 Let $R$ be a 1-dimensional ring, $\bar{R}$ the normalization of $R$ and $C$ the conductor ideal of the extension $R \subset \bar{R}$. Assume $\bar{R} / C=R / C+a_{1} R / C+\cdots+a_{m} R / C$, where $a_{i} \in \sqrt{C}$ the radical ideal of $C$ in $\bar{R}$. Let $M$ be a monoid and write $A=\bar{R} / C$.

(i) If $\sigma \in \mathrm{SL}_{n}(A[M])$, then $\sigma=\sigma_{1} \sigma_{2}$, where $\sigma_{1} \in \mathrm{SL}_{n}((R / C)[M])$ and $\sigma_{2} \in \mathrm{E}_{n}(A[M])$.

(ii) If $P$ is a projective $R[M]$-module of rank $r$, then $P \cong \wedge^{r} P \oplus R[M]^{r-1}$.

Proof (i) Let $\sigma=\left(b_{i j}\right) \in \mathrm{SL}_{n}(A[M])$. Write $b_{i j}=\left(b_{i j}\right)_{0}+\left(b_{i j}\right)_{1} a_{1}+\cdots+\left(b_{i j}\right)_{m} a_{m}$, where $\left(b_{i j}\right)_{l} \in$ $(R / C)[M]$. If $\alpha=\left(\left(b_{i j}\right)_{0}\right)$, then $\operatorname{det}(\sigma)=1=\operatorname{det}(\alpha)+c$, where $c \in(\sqrt{C} / C)[M]$. Since $c \in(R / C)[M]$ is nilpotent, $\operatorname{det}(\alpha)$ is a unit in $(R / C)[M]$. Let $\beta=\operatorname{diagonal}(1 /(1-c), 1, \ldots, 1) \in \mathrm{GL}_{n}((R / C)[M])$ and $\sigma_{1}=\alpha \beta \in \mathrm{SL}_{n}((R / C)[M])$.

Note that $\sigma_{1}^{-1} \sigma=\beta^{-1} \alpha^{-1} \sigma=\beta^{-1} 1 /(1-c) \bar{\alpha} \sigma$, where $\bar{\alpha}=\left(\left(\bar{b}_{i j}\right)_{0}\right),\left(\bar{b}_{i j}\right)_{0}$ are minors of $\left(b_{i j}\right)_{0}$.

$$
\sigma_{2}:=\sigma_{1}^{-1} \sigma=\left[\begin{array}{cccc}
1 & 0 & \cdots & 0 \\
0 & \frac{1}{1-c} & \cdots & 0 \\
\vdots & \vdots & \cdots & \vdots \\
0 & 0 & \cdots & \frac{1}{1-c}
\end{array}\right]\left[\begin{array}{cccc}
1+c_{11} & c_{12} & \cdots & c_{1 n} \\
c_{21} & 1+c_{22} & \cdots & c_{2 n} \\
\vdots & \vdots & \cdots & \vdots \\
c_{n 1} & c_{n 2} & \cdots & 1+c_{n n}
\end{array}\right]
$$

where $c_{i j} \in(\sqrt{C} / C)[M]$

Note that $\sigma_{2} \in \mathrm{SL}_{n}(A[M])$ and $\sigma_{2}=I d$ modulo the nilpotent ideal of $A[M]$. Hence $\sigma_{2} \in \mathrm{E}_{n}(A[M])$. Thus we get $\sigma=\sigma_{1} \sigma_{2}$ with the desired properties.

(ii) Follow the proof of (3.13) and use (3.15)(i)) to get the result.

Now we prove (1.6(ii)) which follows from (3.14) and (3.15).

Theorem 3.16 Let $R$ be a 1-dimensional uni-branched affine algebra over an algebraically closed field and $M$ a monoid. If $P$ is a projective $R[M]$-module of rank $r$, then $P \cong \wedge^{r} P \oplus R[M]^{r-1}$.

\section{Applications}

Let $R$ be a ring of dimension $d$ and $Q$ a finitely generated $R$-module. Let $\mu(Q)$ denote the minimum number of generators of $Q$. By Forster [7] and Swan [22], $\mu(Q) \leq \max \left\{\mu\left(Q_{\mathfrak{p}}\right)+\operatorname{dim}(R / \mathfrak{p}) \mid \mathfrak{p} \in\right.$ $\left.\operatorname{Spec}(R), Q_{\mathfrak{p}} \neq 0\right\}$. In particular, if $P$ is a projective $R$-module of rank $r$, then $\mu(P) \leq r+d$.

The following result is well known.

Theorem 4.1 Let $A$ be a ring such that Serre $\operatorname{dim} A \leq d$. Assume $A^{m}$ is cancellative for $m \geq d+1$. If $P$ is a projective A-module of rank $r \geq d+1$, then $\mu(P) \leq r+d$. 
Proof Assume $\mu(P)=n>r+d$. Consider a surjection $\phi: A^{n} \rightarrow P$ with $Q=\operatorname{ker}(\phi)$. Then $A^{n} \cong P \oplus Q$. Since $Q$ is a projective $A$-module of rank $\geq d+1, Q$ has a unimodular element $q$. Since $\phi(q)=0, \phi$ induces a surjection $\bar{\phi}: A^{n} / q A^{n} \rightarrow P$. Since $n-1>d, A^{n-1}$ is cancellative. Hence $A^{n-1} \cong A^{n} / q A$ and $P$ is generated by $n-1$ elements, a contradiction.

The following result is immediate from (4.1, 3.4, 3.6 and [6]).

Corollary 4.2 Let $R$ be a ring of dimension $d, M$ a monoid and $P$ a projective $R[M]$-module of rank $r>d$. Then:

(i) If $M \in \mathcal{C}(\Phi)$, then $\mu(P) \leq r+d$.

(ii) If $M \subset \mathbb{Z}_{+}^{2}$ is a normal monoid of rank 2 , then $\mu(P) \leq r+d$.

Let $M$ be a $c$-divisible monoid, $R$ a ring of dimension $d$ and $n \geq \max \{2, d+1\}$. Then Schaubhüser [19] proved that $E_{n+1}(R[M])$ acts transitively on $\mathrm{Um}_{n+1}(R[M])$. Using Schaubhüser's result and arguments of Dhorajia-Keshari ([6], Theorem 4.4), we get that if $P$ is a projective $R[M]$-module of rank $n$, then $E(R[M] \oplus P)$ acts transitively on $\operatorname{Um}(R[M] \oplus P)$. Therefore the following result is immediate from 4.1 and 3.13 .

Corollary 4.3 Let $R$ be a ring of dimension $1, M$ a c-divisible monoid and $P$ a projective $R[M]$ module of rank $r \geq 3$. Then $\mu(P) \leq r+1$.

Acknowledgement. We would like to thank the referee for his/her critical remark. The second author would like to thank C.S.I.R., India for their fellowship.

\section{References}

[1] D.F. Anderson, Projective modules over subrings of $k[X, Y]$ generated by monomials, Pacific J. Math. 79 (1978) 5-17.

[2] S.M. Bhatwadekar, Inversion of monic polynomials and existence of unimodular elements (II), Math. Z. 200 (1989) 233-238.

[3] S.M. Bhatwadekar, H. Lindel and R.A. Rao, The Bass-Murthy question: Serre dimension of Laurent polynomial extensions, Invent. Math. 81 (1985) 189-203.

[4] S.M. Bhatwadekar and A. Roy, Some theorems about projective modules over polynomial rings, J. Algebra 86 (1984) 150-158.

[5] W. Bruns and J. Gubeladze, Polytopes, Rings and K-Theory, Springer Monographs in Mathematics, 2009.

[6] A.M. Dhorajia and M.K. Keshari, A note on cancellation of projective modules, J. Pure and Applied Algebra 216 (2012) 126-129.

[7] Otto Forster, Über die Anzahl der Erzeugenden eines Ideals in einem Noetherschen Ring, Math. Z. 84 (1964) 80-87.

[8] J. Gubeladze, Anderson's conjecture and the maximal class of monoid over which projective modules are free, Math. USSR-Sb. 63 (1988), 165-188. 
[9] J. Gubeladze, Classical algebraic K-theory of monoid algebras, Lect. Notes Math. 1437 (1990), Springer, 36-94.

[10] J. Gubeladze, The elementary action on unimodular rows over a monoid ring, J. Algebra 148 (1992) 135-161.

[11] J. Gubeladze, K-Theory of affine toric varieties, Homology, Homotopy and Appl. 1 (1999) 135-145.

[12] M.C. Kang, Projective modules over some polynomial rings, J. Algebra 59 (1979) 65-76.

[13] H. Lindel, Unimodular elements in projective modules, J. Algebra 172 (1995) no-2, 301-319.

[14] B. Plumstead, The conjectures of Eisenbud and Evans, Amer. J. Math. 105 (1983) 1417-1433.

[15] D. Popescu. On a question of Quillen, Bull. Math. Soc. Sci. Math. Roumanie (N.S.) 45 (93) no. 3-4, (2002) 209-212.

[16] D. Quillen. Projective modules over polynomial rings, Invent. Math. 36 (1976), 167-171.

[17] A. Roy, Application of patching diagrams to some questions about projective modules, J. Pure Appl. Algebra 24 (1982), no. 3, 313-319.

[18] H.P. Sarwar, Some results about projective modules over monoid algebras, to appear in Communications in Algebra.

[19] G. Schabhüser, Cancellation properties of projective modules over monoid rings, Universitt Münster, Mathematisches Institut, Münster, (1991) iv+86 pp.

[20] J.P. Serre, Sur les modules projectifs, Sem. Dubreil-Pisot 14 (1960-61) 1-16.

[21] A.A. Suslin, Projective modules over polynomial rings are free, Sov. Math. Dokl. 17 (1976), 1160-1164.

[22] R.G. Swan, The number of generators of a module, Math. Z. 102 (1967), 318-322.

[23] R.G Swan, Gubeladze proof of Anderson's conjecture, Contemp. Math 124 (1992), 215-250. 